\title{
Urgences
}

\section{Liminaire: Ordinateur et Création Textuelle : Écritures et théories}

\section{Marie Bélisle}

Numéro 22, janvier 1989

Octet

URI : https://id.erudit.org/iderudit/025500ar

DOI : https://doi.org/10.7202/025500ar

Aller au sommaire du numéro

Éditeur(s)

Urgences

ISSN

0226-9554 (imprimé)

1927-3924 (numérique)

Découvrir la revue

Citer ce document

Bélisle, M. (1989). Liminaire: Ordinateur et Création Textuelle : Écritures et théories. Urgences, (22), 5-8. https://doi.org/10.7202/025500ar d'utilisation que vous pouvez consulter en ligne.

https://apropos.erudit.org/fr/usagers/politique-dutilisation/ 


\section{LIMINAIRE}

\section{Ordinateur et Création Textuelle: ÉCRITURES ET THÉORIES}

Que l'on s'entende bien: l'ordinateur n'est pas un gadget miracle pour écrivains «branchés»; |'ordinateur n'écrit pas à la place de l'écrivain; l'ordinateur n'empêche pas l'écrivain d'écrire; l'ordinateur n'est pas une lampe d'Aladin d'où le génie s'échappe; l'ordinateur est une machine perfectionnée (et perfectible) qui peut être mise au service de l'écriture.

Depuis longtemps (dans le domaine de l'informatique, le temps passe vite...), les logiciels de traitement de texte permettent aux scripteurs de gérer la production ou la transcription de leurs textes (saisie, corrections et modifications de tous ordres, copie, etc.); depuis longtemps, des informaticiens, des linguistes, des écrivains poursuivent des recherches sur les manipulations de texte rendues possibles par l'informatique. Les développements technologiques récents (augmentation de l'espace-mémoire, raffinement des langages de programmation, etc.) et l'accroissement de l'accessibilité aux systèmes informatisés qui en découle permettent aujourd'hui un travail textuel qui dépasse, de loin, les déplacements de paragraphes et la production automatisée de cadavres exquis. L'idée d'utiliser l'ordinateur comme «assistant", dans les opérations d'écriture, n'est déjà plus nouvelle... Mais le travail se poursuit, le champ des applications de l'informatique s'agrandit et, en même temps, se diversifient les pratiques et les réflexions théoriques qui y sont reliées.

Urgences ne prétend pas, par la publication de ce numéro, faire le tour de la question; Urgences désire seulement ajouter (un peu) aux ouvrages et articles qui, déjà, l'ont abordée et permettre à ceux et celles qui pratiquent la littérature assistée par ordinateur de livrer les résultats de leurs recherches les plus récentes.

Au sommaire, dans le désordre:

- Éléments de bibliographie commentée, une liste for- 
cément incomplète d'ouvrages et articles questionnant les rapports de l'écriture et de l'informatique;

- Mémoire vive, des réflexions, commentaires, exemples, etc. (tirés de textes présentés en bibliographie) disséminés dans le numéro, entre les articles;

- M.O.T.A.M.O., les Manipulations Originales de Textes Assistées par Micro-Ordinateur de Bernard Magné;

- Obscures Constructions, ou les Tentatives et Expériimentations Timides d'une novice (Marie Bélisle)...

- Pastiches, un article de Louise Proulx accompagné de textes produits par des étudiants dans le cadre d'un atelier d'écriture qu'elle animait;

- Urgence, une nouvelle de Jean-Pierre Balpe, produite par le logiciel ROMAN dont il est l'auteur.

L'Objectif: faire Connaître les Tendances actuelles de la recherche (productions Expérimentales et avancées Théoriques), dans le domaine du traitement ${ }^{\star}$ de(s) texte(s) par ordinateur. Donc faire voir, par la présentation des travaux d'écrivains et d'informaticiens, comment l'ordinateur sert l'écriture.

MARIE BELISLE

*STRICTO SENSU: «manière de traiter (une substance); opération, procédé permettant de modifier (une matière), (Petit Robert, 1987). 


\section{MÉMOIRE VIVE}

En aucun cas, s'agissant d'écriture, l'ordinateur ne permet l'économie d'une théorie, d'une pratique et d'une pédagogie du texte. Mais en chacun de ces domaines, l'informatique peut jouer un rôle important. À une condition: qu'elle fasse elle-même l'objet, dans son rapport au texte, d'une pédagogie, d'une pratique et d'une théorie.

Bernard Magné, $5^{\star}$, p. 4

*Les chiffres de référence utilisés dans cette section renvoient, systématiquement, à la numérotation des textes présentés dans les Éléments de bibliographie commentée. 


\section{MÉMOIRE VIVE}

La machine-auteur? Pourquoi pas, si le jugement est à l'aune de la production d'un texte, d'une oeuvre, littéralement inédits. Mais cette «originalité» qui pourrait peut-être se défendre au regard des critères traditionnels n'est que la manifestation de l'extraordinaire fécondité combinatoire que recèle tout modèle. Comme pour la musique, l'auteur n'est-il pas plutôt celui ou ceux qui ont écrit la partition (le modèle); l'interprète, celui ou ceux, avec ou sans talent, qui lancent l'exécution du programme, en fournissant les données qui permettront des réalisations particulières (et probablement inédites en effet) du modèle?

Mario Borillo, 6, p. 240 